\title{
Stem cell therapy in the aging hearts of Fisher 344 rats: Synergistic effects on myogenesis and angiogenesis
}

\author{
Jiang-Yong Min, MD, ${ }^{a}$ Yu Chen, MD, ${ }^{a}$ Sohail Malek, BS, ${ }^{a}$ Achim Meissner, MD, ${ }^{\text {b }}$ Meixiang Xiang, MD, PhD, ${ }^{a}$ \\ Qinen Ke, MD, ${ }^{a}$ Xin Feng, MD, ${ }^{a}$ Masaharu Nakayama, MD, PhD, ${ }^{a}$ Emel Kaplan, MD, and James P. Morgan, MD, PhD ${ }^{a}$
}

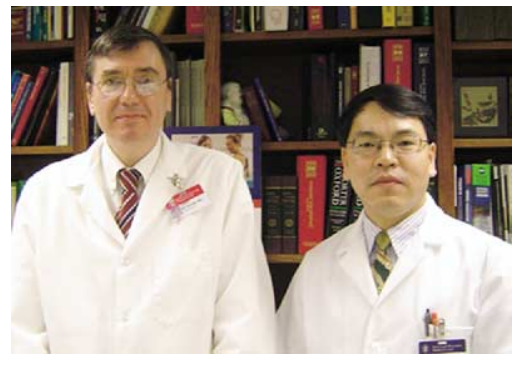

Morgan and Min
From the Cardiovascular Division, ${ }^{\mathrm{a}}$ Beth Israel Deaconess Medical Center and Harvard Medical School, Boston, Mass, and the Department of Cardiology, ${ }^{\mathrm{b}}$ University of Kiel, Kiel, Germany.

Supported in part by National Institutes of Health Grant R01 DA-12774 (Dr Morgan) and National Institute of Aging Grant 5P60 AG08814-13 (Dr Min). Dr Xiang (the 2nd affiliated Hospital, Medical College of Zhejiang University, China) is the recipient of a scholarship from the China Scholarship Council.

Received for publication March 23, 2004; revisions received Nov 17, 2004; accepted for publication March 8, 2005.

Address for reprints: James P. Morgan, MD, $\mathrm{PhD}$, Cardiovascular Division, Beth Israel Deaconess Medical Center, 330 Brookline Ave, Boston, MA 02215 (E-mail: jmorgan@ bidmc.harvard.edu).

J Thorac Cardiovasc Surg 2005; 130:547-53

0022-5223/\$30.00

Copyright (C) 2005 by The American Association for Thoracic Surgery

doi:10.1016/j.jtcvs.2005.03.017
Objective: Advanced age is a major risk factor for ventricular dysfunction and reduction of cardiac reserve. Finding novel approaches to prevent and attenuate heart dysfunction associated with advanced age is a major therapeutic challenge. The present study was designed to test whether engrafted embryonic stem cells could improve myocardial function in aging hearts.

Methods: Cultured mouse embryonic stem cells used for cell therapy were transfected with green fluorescent protein. Aging rats in the cell-treated group received intramyocardial injection of embryonic stem cells. Hemodynamic measurement, myocyte counting, and evaluation of blood flow were performed 6 weeks after cell transplantation.

Results: Embryonic stem cell therapy partially improved cardiac reserve, as reflected by the in vivo response to isoproterenol (INN: isoprenaline) stimulation in aging hearts 6 weeks after cell implantation. The functional benefits from engrafted embryonic stem cells were associated with increased myocyte numbers and enhanced left ventricular blood perfusion in the aging heart. The characteristic phenotype of engrafted embryonic stem cells was identified in the transplanted heart on the basis of green fluorescent protein-positive spots that were further demonstrated to differentiate into cardiac tissue with positive staining for cardiac $\alpha$-myosin heavy chain.

Conclusions: Regenerating cardiomyocytes and increasing regional blood perfusion in the aging heart after embryonic stem cell transplantation synergistically resulted in improvement of cardiac function. Embryonic stem cell transplantation might hold significant clinical potential in attenuating the progressive decrease of cardiac function associated with advanced aging.

$\mathrm{E}$ pidemiologic studies have shown that congestive heart failure is the major cause of most hospitalizations of persons older than 65 years. ${ }^{1,2}$ Heart transplantation is an acceptable alternative for treating end-stage heart failure in addition to drug treatment; however, only a small number of patients can receive heart transplantation because of the shortage of donors. Therefore finding novel and effective approaches to prevent and reduce the pathogenesis of heart dysfunction associated with advancing age is a major therapeutic challenge.

It has been demonstrated through the last decade that the aging process of the heart in animals ${ }^{3,4}$ and human subjects ${ }^{5}$ is characterized by a significant loss of cardiac myocytes, especially in the left ventricle. A recent study ${ }^{6}$ documented that aging hearts have impaired angiogenic function as a result of decreased production of a platelet-derived growth factor. Impaired angiogenesis in aging hearts might facilitate the myocyte loss and subsequently reduce heart function. Recently, therapeutic approaches aimed at promoting angiogenesis and regenerating new cardiac myocytes have been undergoing intensive investigation. Engrafted cells have been 
shown to survive, proliferate, and form gap junctions with the host myocardium ${ }^{7,8}$ and partially restore the impaired cardiac function in either cryoinjured ${ }^{9}$ or infarcted ${ }^{10}$ hearts. Our recent studies demonstrated that embryonic stem (ES) cells can differentiate into cardiac-like cells and improve cardiac function in postinfarcted rat hearts at 6 weeks ${ }^{11}$ and 32 weeks $^{12}$ after cell implantation. The present study was designed to determine whether ES cells after intramyocardial implantation could regenerate new cardiac myocytes and enhance cardiac function in aging hearts.

\section{Materials and Methods \\ ES Cell Preparation and Transplantation}

The mouse ES cell line ES-D3 was purchased from the American Type Culture Collection (ATCC, Manassas, Va) and cultured with a previously described method. ${ }^{11,12}$ Before transplantation, cells dissected from beating clusters were transfected with green fluorescent protein (GFP), a marker for the identification of engrafted cells. Two days after GFP transfection, cultured ES cells were trypsinized and resuspended in Joklik modified medium (Sigma, St Louis, Mo) with a density of $2 \times 10^{7}$ cells $/ \mathrm{mL}$ for cell transplantation.

Experiments were performed in 46 senescent male Fisher 344 rats aged 24 months (obtained from the National Institute on Aging) and 24 adult male Fisher 344 rats aged 3 months (Charles River, Wilmington, Mass). The investigation conformed to the "Guide for the Care and Use of Laboratory Animals" published by the US National Institutes of Health (National Institutes of Health publication no. 85-23, revised 1996), and the protocol was approved by our institutional animal care committee. Animals were intubated after achievement of anesthesia with pentobarbital (60 $\mathrm{mg} / \mathrm{kg}$ administered intraperitoneally) and ventilated with room air by using a small animal ventilator (Harvard Apparatus, South Natick, Mass). Intramyocardial injection of the ES cell suspension (50 $\mu \mathrm{L}$, approximately $10^{6}$ cells) was performed in 3 different sites ( 1 in the apex and 2 in the middle of the left ventricle) with a tuberculin syringe. The adult and aging control rats received the exact volume of the cell-free medium. Two additional aging rats receiving stem cell injection were used for monitoring cardiac arrhythmia. The electrocardiographic (ECG) transmitter (Data Sciences International Inc, St Paul, Minn) was implanted in the rat abdominal cavity with a previously described method, ${ }^{13}$ and the signal was collected by a receiver (Data Sciences International Inc).

\section{Functional Measurement and $\boldsymbol{\beta}$-adrenergic Responsiveness In Vivo}

Rats (10 for each group) were anesthetized with pentobarbital (60 $\mathrm{mg} / \mathrm{kg}$ administered intraperitoneally) at 6 weeks after cell transplantation. Hemodynamic measurements in vivo were performed with a modified method, as described previously. ${ }^{11,12}$ After hemodynamic measurements at baseline, serially increasing intravenous bolus injections of isoproterenol were given in doses of $0.1,0.3$, $1.0,3.0$, and $6.0 \mu \mathrm{g} / \mathrm{kg}$ through the femoral vein to determine the inotropic responsiveness to $\beta$-adrenergic stimulation. The doses of isoproterenol used in the present study were chosen on the basis of a previous report. ${ }^{14}$ Hemodynamic responses to $\beta$-adrenergic stim- ulation were collected at 8 - to 10-minute intervals to obtain a dose-response curve.

\section{Regional Blood Flow Measurement and Numeric Density of Arterioles}

Stable, nonradioactive isotope-labeled microspheres (15 $\mu \mathrm{m}$; BioPAL Inc, Worcester, Mass) were used to determine the regional blood flow in anesthetized rats 20 minutes after measurement of hemodynamics and $\beta$-adrenergic stimulation. The method was modified from a previous publication. ${ }^{15}$ In brief, a set of microspheres $\left(1.25 \times 10^{6}\right.$ in $\left.0.5 \mathrm{~mL}\right)$ was diluted in $0.5 \mathrm{~mL}$ of sanSaline saline (BioPAL Inc) and injected into the left atrium over 10 seconds during cardiac surgery. Reference blood samples were withdrawn by using a syringe at a constant rate of 2-minute intervals through the femoral artery, resulting in a 2-mL sample used to calculate absolute myocardial blood flow. The rat heart was then harvested during deep anesthesia. The heart was weighed and normalized by body weight. The left ventricle was surgically isolated and cut into transmural slices. Two segments from the middle of the left ventricle and one from the apex were used for blood flow measurement. The average myocardial sample weighed approximately $0.15 \mathrm{~g}$. A piece of the right ventricle was isolated for tissue control reference. Finally, the tissue and blood samples were shipped to BioPAL Inc for measurement of active isotope microspheres and determination of myocardial regional blood flow.

The numeric density of arterioles (diameter $>20 \mu \mathrm{m}$ ) was counted in each area observed (5 for each group) on hematoxylin and eosin-stained slides by using a light microscope at $400 \times$ magnification. Five high-power fields in the transplanted area were randomly selected; the number of arterioles in each section was averaged and expressed as the number of arterioles per unit area (in square millimeters).

\section{Isolated Myocytes and Myocyte Number Counting}

In another set of experiments (7 adult control, 6 aging control, and 6 aging rats with cell injection), rats were anesthetized with pentobarbital 6 weeks after cell transplantation. The procedure of myocyte isolation was modified from a method described previously. ${ }^{16}$ Isolated myocytes were counted with a hemocytometer using a microscope. Hemodynamics were measured as described above before dissecting cardiomyocytes for linear regression study.

\section{Identification of Transplanted Cells, Histologic Analysis, and Western Blotting}

Five additional rats for each group were used for histologic study. The hearts were harvested after achievement of anesthesia and subsequently dissected into 4 transverse sections from apex to base. Five-micrometer transverse slices from each section were prepared for hematoxylin and eosin staining. The survival of engrafted cells was identified on the basis of GFP-positive clusters from the aging hearts with stem cell injection. Transformation of cardiac-like cells from engrafted ES cells was verified by means of antibody immunostaining for cardiac-specific $\alpha$-myosin heavy chain $(\alpha-\mathrm{MHC})$. Briefly, prepared sections were incubated with a mouse anti- $\alpha$-MHC monoclonal antibody (Berkeley Antibody Co, Richmond, Calif) for 60 minutes at room temperature. After wash- 
TABLE 1. General characteristics of adult rats and aging rats with or without stem cell therapy

\begin{tabular}{lccc}
\hline & Adult & Aging & $\begin{array}{c}\text { Aging }+ \\
\text { ES cells }\end{array}$ \\
\hline $\mathrm{BW}(\mathrm{g})$ & $352 \pm 10$ & $413 \pm 15$ & $424 \pm 22$ \\
$\mathrm{HW}(\mathrm{g})$ & $0.9 \pm 0.1$ & $1.3 \pm 0.2$ & $1.2 \pm 0.3$ \\
$\mathrm{HW} / \mathrm{BW}^{*} 100$ & $0.2 \pm 0.02$ & $0.3 \pm 0.03$ & $0.3 \pm 0.04$ \\
$\mathrm{HR}(\mathrm{beats} / \mathrm{min})$ & $360 \pm 28$ & $372 \pm 29$ & $375 \pm 26$ \\
LVSP (mm Hg) & $126 \pm 15$ & $108 \pm 11$ & $112 \pm 10$ \\
LVEDP (mm Hg) & $5.8 \pm 0.7$ & $11.2 \pm 1.6 \dagger$ & $8.4 \pm 1.1^{*} \ddagger$ \\
$+\mathrm{dP} / \mathrm{dtmax}(\mathrm{mm} \mathrm{Hg})$ & $10,296 \pm 954$ & $9218 \pm 806$ & $9626 \pm 915$ \\
$-\mathrm{dP} / \mathrm{dtmax}(\mathrm{mm} \mathrm{Hg})$ & $9329 \pm 870$ & $8017 \pm 475^{*}$ & $8798 \pm 520 \ddagger$ \\
\hline
\end{tabular}

Values are presented as means \pm SD. Measurements were conducted at 6 weeks after ES cell or medium injection in 10 rats for each group. $B W$, Body weight; $H W$, heart weight; $H W / B W$, ratio of heart weight/body weight; $H R$, heart rate; $L V S P$, left ventricular systolic pressure; $L V E D P$, left ventricular end-diastolic pressure; $+d P / d t m a x$, rate of peak left ventricular systolic pressure increase; $-d P / d t m a x$, rate of peak left ventricular systolic pressure decrease. $* P<.05$ and $\dagger P<.01$ versus adult group; $\ddagger P<$ .05 , aging + ES cell group versus aging group.

ing with PBS, sections were incubated with rabbit anti-goatconjugated rhodamine IgG. Immunostaining was performed on serial sections of the rat heart. Furthermore, the protein levels of sarcoplasmic reticulum $\mathrm{Ca}^{2+}$ ATPase (SERCA2) were measured in additional left ventricles ( 5 for each group) with a previously described method ${ }^{17}$ to evaluate the activity of cardiac protein.

\section{Statistical Analysis}

All data are presented as means $\pm \mathrm{SD}$. Differences between groups were evaluated by means of 1-way analysis of variance. Means shown to be different between individual groups were compared with paired or unpaired Student $t$ tests. The results of isoproterenol stimulation were analyzed by using 2-way analysis of variance. Linear regression modeling was used to compare parameters of the left ventricular systolic pressure (LVSP) and regional blood flow and parameters of the LVSP and the number of single isolated myocytes.

\section{Results}

\section{General Characteristics and Ventricular Function} After ES Cell Therapy

Three aging control rats with significant weight loss in the follow-up period before death were excluded. No tumors were seen during necropsy at the end of the study in any animals. During the follow-up period, we did not find significant cardiac arrhythmias with the telemetric ECG monitoring (data not shown). Our data indicated decrease of cardiac function in the aging hearts (Table 1) reflected by a mild reduction of the LVSP, the peak rate of the LVSP increase $(+\mathrm{dP} / \mathrm{dtmax})$, and the peak rate of the LVSP decrease $(-\mathrm{dP} / \mathrm{dtmax})$. However, the left ventricular enddiastolic pressure was significantly increased in aging rats compared with that seen in adult rats. Six weeks after intramyocardial injection of ES cells, the ventricular func-
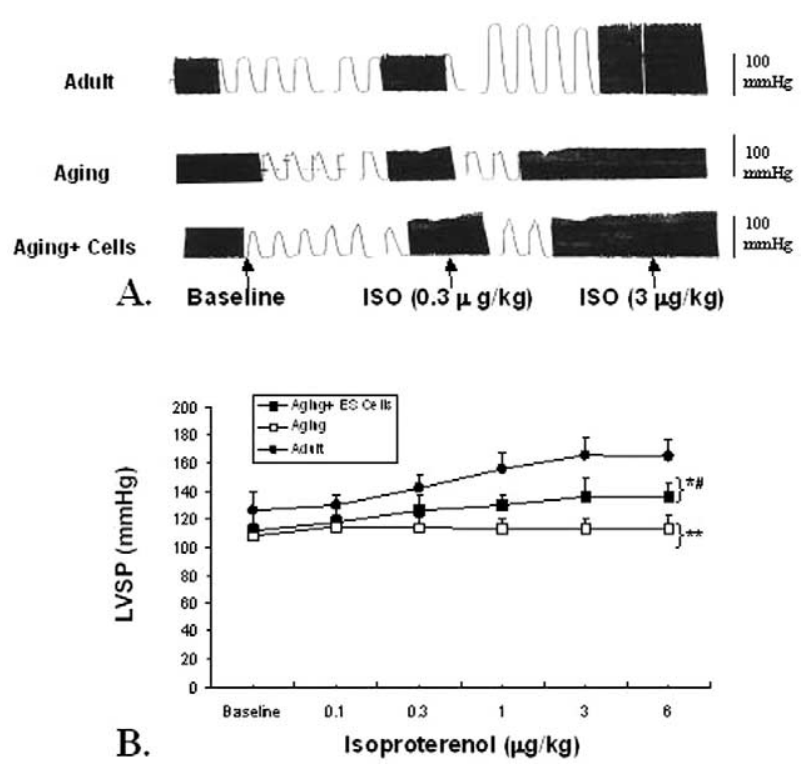

Figure 1. Inotropic response of the LVSP to isoproterenol (ISO) stimulation during hemodynamic measurements in vivo. Original representative recordings $(A)$ show the response to isoproterenol stimulation was blunted in the aging heart without cell treatment. ES cell transplantation partially restored the inotropic response to $\beta$-adrenergic stimulation 6 weeks after cell injection. Summarized data are shown in panel $B .{ }^{*} P<.05$ and ${ }^{* *} P<.01$ versus adult group; $\# P<.05$, aging + ES cell group versus aging group.

tion was improved, accompanied by significantly reduced left ventricular end-diastolic pressure (Table 1). Isoproterenol stimulation induced a dose-dependent increase in the LVSP (Figure 1) in adult control rats, whereas this positive inotropic effect was markedly blunted in the aging heart. ES cell transplantation partly restored the inotropic response to isoproterenol in aging rats 6 weeks after cell injection (Figure 1).

\section{Left Ventricular Blood Flow and Myocyte Regeneration After ES Cell Therapy}

The regional blood flow measured by using stable, nonradioactive isotope microspheres was significantly decreased in the aging heart without cell treatment (Figure 2, A) compared with that seen in the adult heart. Cell transplantation significantly increased the left ventricular myocardial blood perfusion compared with that seen in the aging heart without cell injection. Regression analysis revealed no significant linear relationship $(P=.51)$ between the LVSP and the left ventricular blood flow (Figure 2, B). Additionally, the number of arterioles increased significantly in the aging heart after ES cell transplantation compared with that seen in the aging heart without cell therapy $\left(7.0 \pm 1.3\right.$ arterioles $/ \mathrm{mm}^{2}$ in the aging plus ES cell group vs $5.2 \pm 0.7$ arterioles $/ \mathrm{mm}^{2}$ in 

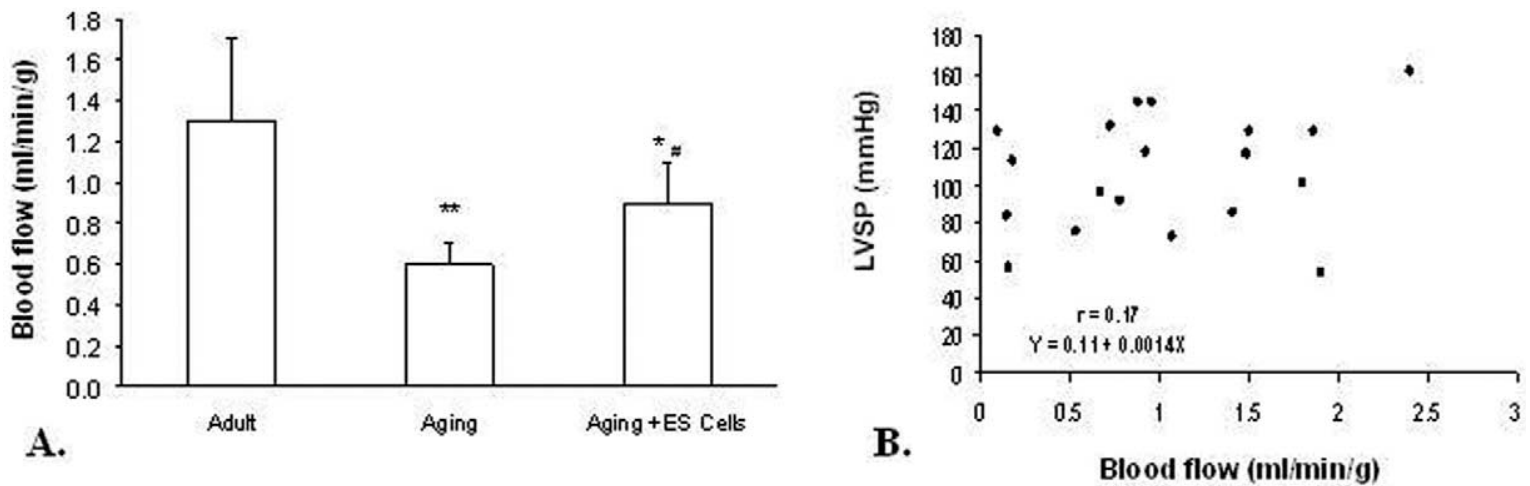

Figure 2. The results of the left ventricular blood flow measurements with isotope microspheres are shown (A). ES cell transplantation significantly restored the regional blood flow compared with the aging heart without cell treatment. A linear regression study (B) demonstrated a weak association between the LVSP and the regional blood flow. ${ }^{*} P<.05$ and ${ }^{* *} P<.01$ versus adult group; $\# P<.05$, aging + ES cell group versus aging group.

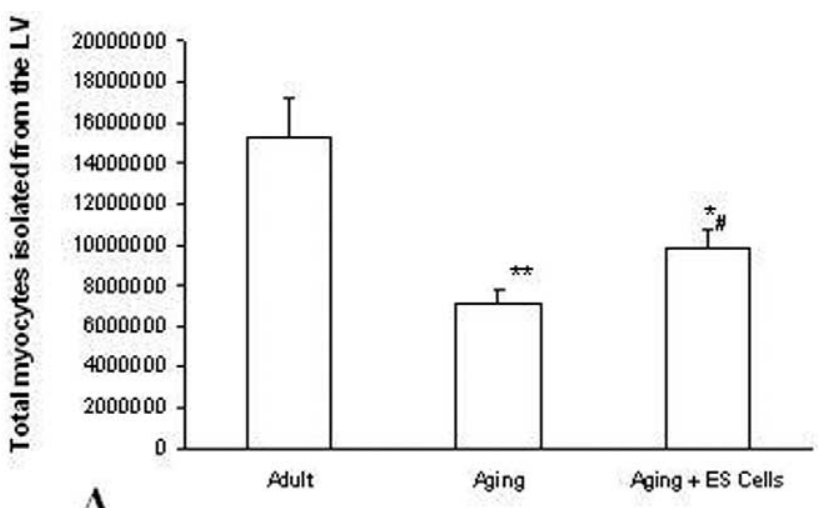

A.

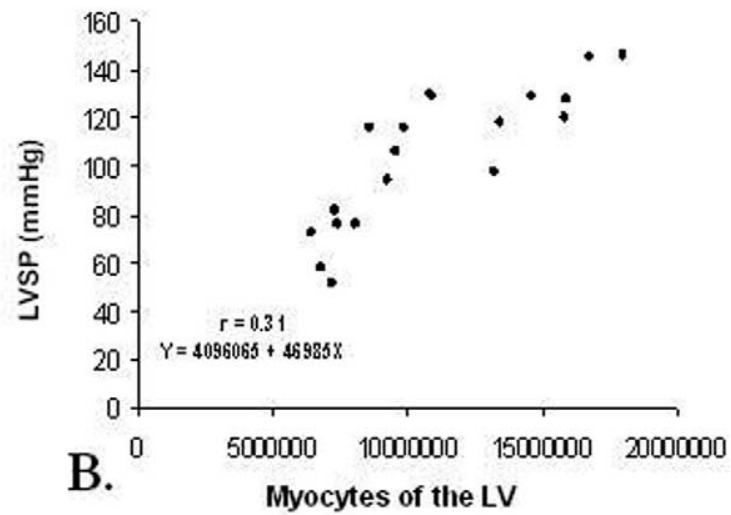

Figure 3. Myocyte counts from the adult and the aging heart with or without cell transplantation (A). The aging heart without cell therapy showed significant loss of cardiac myocytes. ES cell transplantation partially restored the myocyte number toward normal values. A linear regression study $(B)$ demonstrated a moderate association between the LVSP and the number of myocytes. $L V$, Left ventricle. ${ }^{*} \boldsymbol{P}<.05$ and ${ }^{* *} P<.01$ versus adult group; \#P $<.05$, aging + ES cell group versus aging group.

the aging group, $P<.05$ ), although the arteriolar density was still lower than that from the adult heart $(7.0 \pm 1.3$ arterioles $/ \mathrm{mm}^{2}$ in the aging plus ES cell group vs $10.8 \pm$ 1.7 arterioles $/ \mathrm{mm}^{2}$ in the adult group, $P<.05$ ).

The average yields of myocytes in aging left ventricles without ES cell treatment were significantly lower than the number of myocytes yielded from adult rat left ventricles (Figure 3,A). Cell transplantation partially restored the number of myocytes in the aging rat left ventricles compared with that from aging rat left ventricles without cell therapy. Regression analysis showed LVSP and myocyte number to have a slightly positive association, but this did not reach statistical significance (Figure 3, B).
Protein Levels of SERCA2 after ES Cell Therapy, Identification of Implanted ES Cells, and Histologic Study

The protein levels of SERCA2 were analyzed as a ratio compared with reduced glyceraldehyde-phosphate dehydrogenase (GAPDH). As shown in Figure 4, there was a moderate reduction of SERCA2 protein levels in aging left ventricles compared with that from adult left ventricles. ES cell treatment resulted in partially restored protein levels of SERCA2 in left ventricles from aging rats at 6 weeks after cell injection. Figure 5, $A$ and $B$, shows engrafted ES cells distributed within the host myocardium. Those regenerated tissues were not found in either adult myocardium or the aging heart without ES cell injection (data not shown). 
Richly GFP-positive spots (Figure 5, C) were found during fluorescent microscopy in aging myocardium with cell injection. These GFP-positive spots suggest that engrafted ES cells, or at least some of them, survived in the aging myocardium at 6 weeks after cell injection. There was no evidence of overt rejection, which is reflected by a lack of encapsulation and an absence of significant lymphocyte infiltration. The aging heart with cell transplantation showed positive staining to cardiac-specific $\alpha$-MHC (Figure $5, D)$, including the area of newly regenerated cardiac tissue. Colocalization of engrafted ES cells with fluorescent staining for $\alpha$-MHC is shown in Fig 5, E. These data suggest that engrafted ES cells not only survived in the aging myocardium 6 weeks after cell injection but also differentiated into cardiac tissue.

\section{Discussion}

Advanced aging is associated with a decrease in cardiac contractility and relaxation. ${ }^{18,19}$ Potential mechanisms include a significant loss of cardiac myocytes in animals ${ }^{3}$ and in human subjects ${ }^{5}$ as part of the normal aging process. Anversa and associates ${ }^{3}$ reported a significant loss of myocytes in the left ventricles after advanced aging. A further study by Kajstura and colleagues ${ }^{4}$ indicated that the progressive increase in apoptotic and necrotic myocyte death in the aging hearts of Fisher 344 rats was associated with the occurrence of ventricular dysfunction, which was apparent between 16 and 24 months of age.

The results of the present study clearly demonstrate a significant myocyte loss in aging hearts from Fischer 344 rats at the age of 24 months compared with that seen in the hearts of 3-month-old rats. ES cell therapy at 6 weeks after transplantation restored the number of myocytes toward normal values. The survival of engrafted ES cells was confirmed by the finding of GFP-positive tissue in the aging myocardium 6 weeks after cell transplantation, which also had differentiated into cardiac tissue, as reflected by positive staining to cardiac-specific $\alpha$-MHC. The positive response to $\beta$-adrenergic stimulation was blunted in the aging heart without ES cell treatment but could be partially restored 6 weeks after ES cell transplantation. This finding is consistent with our previous results with isolated papillary muscles in diseased hearts ${ }^{11,17}$ and the results of others ${ }^{20}$ with perfused aging hearts. The abnormal ventricular function and isoproterenol responsiveness in the aging heart might be attributed to the reduction in protein level of SERCA2, which is mainly responsible for the dysfunction of myocardial performance ${ }^{17}$ in infarcted rat hearts. Previous studies showed a reduction in the content and activity of SERCA2 in the aging rat heart ${ }^{21}$ and senescent human myocardium. ${ }^{22}$ Loss of cardiomyocytes in the aging rat heart is the major factor contributing to the reduction of SERCA2 protein levels. Decrease in the protein levels of SERCA2 in the
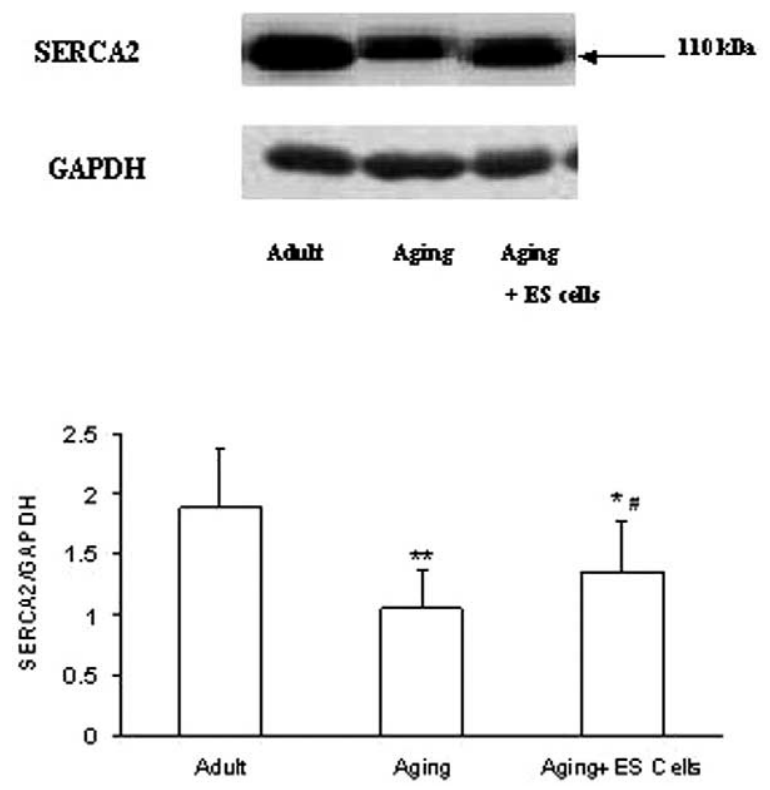

Figure 4. Protein expressions of SERCA2 (5 for each group) in left ventricles from adult rats, aging rats, and aging rats with cell transplantation determined by means of the Western blot technique. A, Western blot; B, densitometric analysis of SERCA2/reduced glyceraldehyde-phosphate dehydrogenase (GAPDH). ${ }^{*} \boldsymbol{P}<.05$ and ${ }^{* *} \boldsymbol{P}<.01$ versus adult group; $\# \boldsymbol{P}<.05$, aging + ES cell group versus aging group.

aging heart resulted in impaired intracellular $\mathrm{Ca}^{2+}$ handling, which is responsible for reduction of cardiac performance and attenuation of the inotropic effect to isoproterenol stimulation. Partial restoration of the inotropic responsiveness to isoproterenol stimulation by ES cell transplantation was related to increased protein levels of SERCA2. More experiments are needed to clarify the effects of ES cell therapy on the expression of major cardiac contraction proteins and intracellular $\mathrm{Ca}^{2+}$ handling in the aging and diseased aging hearts. In addition, $\beta$-receptor downregulation might also evolve into decreased isoproterenol stimulation responsiveness in the aging heart that cannot be excluded without further experimental testing.

Advanced age is accompanied by impaired angiogenesis in the vascular beds of the heart ${ }^{6,23}$ and peripheral tissues ${ }^{24}$ at rest and during ischemic stress. Diminished angiogenesis in old rats and rabbits was the result of impaired endothelial function and a lower expression of vascular endothelial growth factor in nonischemic and ischemic tissue. ${ }^{24}$ With the isotope microsphere techniques, the present study demonstrated a significant reduction of the regional blood perfusion in the aging left ventricles. ES cell transplantation partially restored the regional blood flow of the left ventricle in the aging heart at 6 weeks after cell injection. This finding is consistent with our previous reports that showed stem cell 

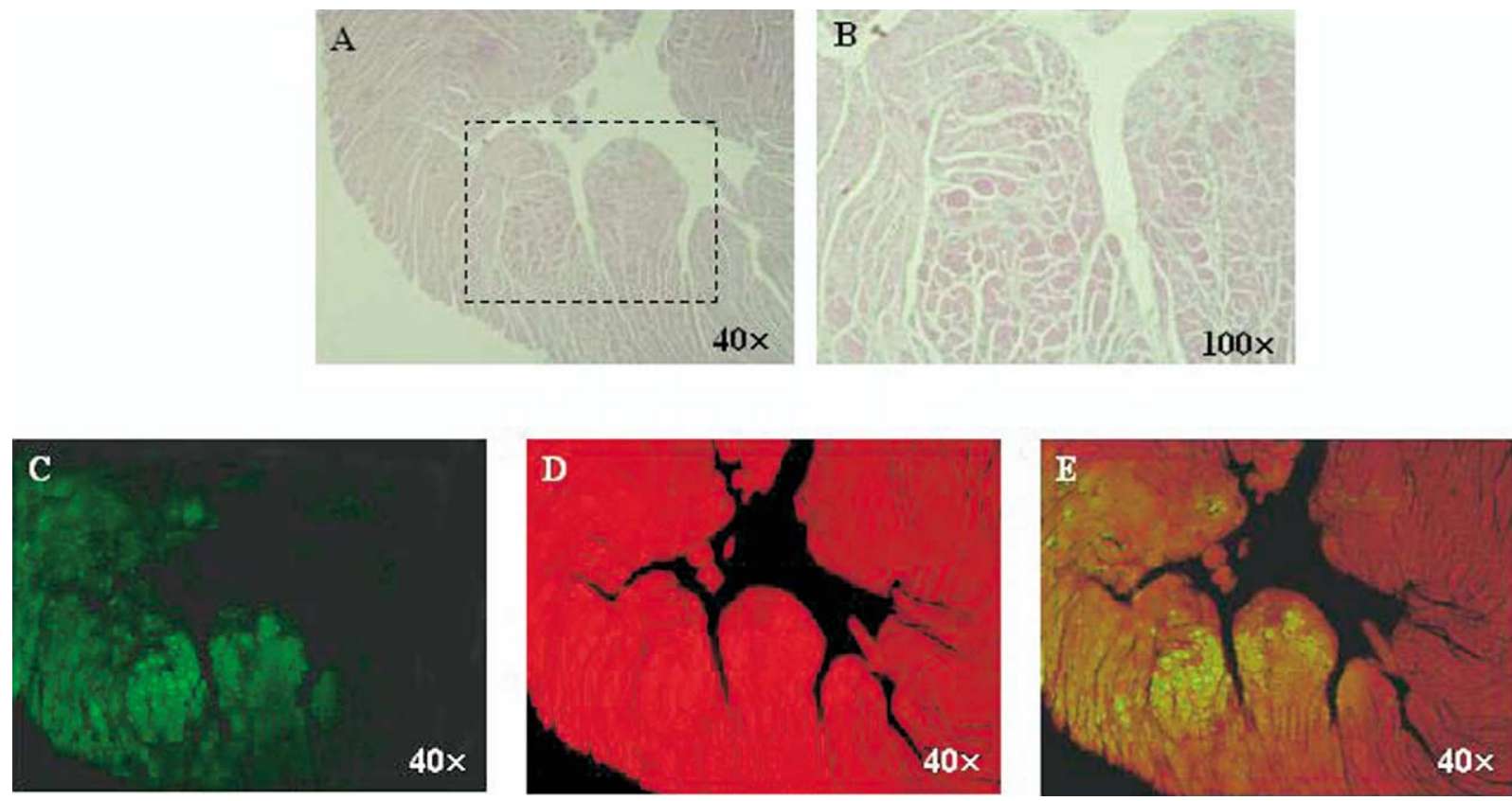

Figure 5. Panel A shows engrafted ES cells with hematoxylin and eosin staining in the aging myocardium 6 weeks after cell transplantation. Panel B shows the higher magnification image corresponding with the image in panel $A$ pointed out by a dot-lined rectangle to show regenerated tissue from implanted ES cells. GFP-positive spots are shown within the aging host myocardium in panel C. The $\alpha$-MHC staining of the myocardial section from the aging heart with cell transplantation, as shown in panel $D$, indicates the positive staining not only displayed in the host myocardium but also in the regenerated tissue. Panel E shows colocalization of transplanted ES cells by using fluorescent staining for cardiac $\alpha$-MHC.

transplantation could enhance angiogenesis by increasing neovascularization in postinfarcted rat myocardium ${ }^{12}$ and increasing the left ventricular blood flow in postinfarcted porcine hearts. ${ }^{15}$ New blood supply to the aging myocardium combined synergistically with regenerating cardiomyocytes derived from engrafted ES cells might prevent and reduce the pathogenesis of ventricular function associated with advancing age. It is possible that transplanted ES cells could release transcription signals (eg, endothelial growth factor) to stimulate neovascularization in the surrounding tissue, or a portion of the engrafted ES cells might transdifferentiate into functional endothelial cells. Further experiments are required to understand the sophisticated mechanisms of blood flow improvement after cell transplantation. The linear regression analyses in the present study do not indicate a strong correlation between a single variable (either myocyte number or blood flow) and ventricular function. No single factor fully contributes all the benefits from cell transplantation in the aging hearts. Myogenesis and angiogenesis from engrafted ES cells provide synergistic effects leading to the improvement of cardiac function.

There are several limitations in the present study. First, cardiac function was measured in the unloaded condition.
Preload, afterload, and heart rate might affect the functional difference between the young and aging hearts. Extensive systematic evaluation of cardiac function under the conditions of different loading conditions should be conducted in a future study.

Second, linear regression analyses might not reflect the natural feature of the host hearts after cell transplantation. The mechanism of cell therapy for cardiac repair is quite complicated and has not been fully addressed yet. Extensive explanation from regression analyses should be done cautiously because even a more complete multivariable regression study might not establish a causal relationship.

Third, myocyte increase after cell transplantation might not only result from engrafted ES cells per se but also from decrease of the apoptotic process in the aging myocardium caused by cardiac-protecting factors that were released from engrafted ES cells. Augmentation of angiogenesis results in an increase in left ventricular blood flow that might also contribute partially to a reduction in the process of apoptosis in the advanced aging heart.

Fourth, although there was no evidence of ventricular arrhythmia in the aging rat heart after ES cell transplanta- 
tion with telemetric ECG monitoring, an extensive study should be performed to test cell therapy-associated arrhythmia before using this novel approach clinically in patients.

\section{References}

1. Haan MN, Selby JV, Quesenberry CP Jr, Schmittdiel JA, Fireman BH, Rice DP. The impact of aging and chronic disease on use of hospital and outpatient services in a large HMO: 1971-1991. J Am Geriatr Soc. 1997;45:667-74

2. Masoudi FA, Havranek EP, Krumholz HM. The burden of chronic congestive heart failure in older persons: magnitude and implications for policy and research. Heart Fail Rev. 2002;7:9-16.

3. Anversa P, Palackal T, Sonnenblick EH, Olivetti G, Meggs LG, Capasso JM. Myocyte cell loss and myocyte cellular hyperplasia in the hypertrophied aging rat heart. Circ Res. 1990;67:871-85.

4. Kajstura J, Cheng W, Sarangarajan R, Li P, Li B, Nitahara JA, et al. Necrotic and apoptotic myocyte cell death in the aging heart of Fisher 344 rats. Am J Physiol. 1996;271:H1215-28.

5. Olivetti G, Melissari M, Capasso JM, Anversa P. Cardiomyopathy of the aging human heart: myocyte loss and reactive cellular hypertrophy. Circ Res. 1991;68:1560-8.

6. Edelberg JM, Lee SH, Kaur M, Tang L, Feirt NM, MaCabe S, et al. Platelet-derived growth factor- $\mathrm{AB}$ limits the extent of myocardial infarction in a rat model: feasibility of restoring impaired angiogeneic capacity in the aging heart. Circulation. 2002;105:608-13.

7. Reinecke H, Zhang M, Bartosek T, Murry CE. Survival, integration, and differentiation of cardiomyocyte grafts: a study in normal and injured rat hearts. Circulation. 1999;100:193-202.

8. Scorsin M, Marotte F, Sabri A, Le Dref O, Demirag M, Samuel JL, et al. Can grafted cardiomyocytes colonize peri-infarction myocardial areas? Circulation. 1996;94(suppl II):II337-40.

9. Li RK, Jia ZQ, Weisel RD, Mickel DAG, Zhang J, Mohabeer MK, et al. Cardiomyocyte transplantation improves heart function. Ann Thorac Surg. 1996;62:654-61.

10. Orlic D, Kajstura J, Chimenti S, Jakoniuk I, Anderson SM, Li B, et al. Bone marrow cells regenerate infarcted myocardium. Nature. 2001; 410:701-5.

11. Min JY, Yang Y, Converso KL, Liu L, Huang Q, Morgan JP, et al. Transplantation of embryonic stem cells improves cardiac function in postinfarcted rats. J Appl Physiol. 2002;92:288-96.
12. Min JY, Yang Y, Sullivan MF, Ke Q, Converso KL, Chen Y, et al. Long-term improvement of cardiac function in rats after infarction by transplantation of embryonic stem cells. J Thorac Cardiovasc Surg. 2003; 125:361-9.

13. Stilli D, Sgoifo A, Macchi E, Zaniboni M, de Lasio S, Cerbai E, et al. Myocardial remodeling and arrhythmogenesis in moderate cardiac hypertrophy in rats. Am J Physiol Heart Circ Physiol. 2001;280:H142-50.

14. Cherng WJ, Liang CS, Hood WB Jr. Effects of metoprolol on left ventricular function in rats with myocardial infarction. Am J Physiol Heart Circ Physiol. 1994;266:H783-94.

15. Min JY, Sullivan MF, Yang Y, Zhang JP, Converso KL, Morgan JP, et al. Significant improvement of heart function by cotransplantation of human mesenchymal stem cells and fetal cardiomyocytes in postinfarcted pigs. Ann Thorac Surg. 2002;74:1568-75.

16. Wolska BM, Solaro RJ. Method for isolation of adult mouse cardiac myocytes for studies of contraction and microfluorimetry. Am J Physiol Heart Circ Physiol. 1996;271:H1250-5.

17. Min JY, Meissner A, Feng X, Wang J, Malek S, Wang JF, et al. Dantrolene: effects on abnormal intracellular $\mathrm{Ca} 2+$ handling and inotropy in postinfarcted rat myocardium. Eur J Pharmacol. 2003;471: 41-7.

18. Lakatta EG. Cardiovascular regulatory mechanisms in advanced age. Physiol Rev. 1993;73:413-67.

19. Wei JY. Age and the cardiovascular system. N Engl J Med. 1992;327: 1735-9.

20. Tao M, Moffat MP, Narayanan N. Age-related alterations in the phosphorylation of sarcoplasmic reticulum and myofibrillar proteins and diminished contractile response to isoproterenol in intact rat ventricle. Circ Res. 1993;72:102-11.

21. Schmidt U, del Monte F, Miyamoto MI, Matsui T, Gwathmey JK, Rosenzweig A, et al. Restoration of diastolic function in senescent rat hearts through adenoviral gene transfer of sarcoplasmic reticulum Ca2+-ATPase. Circulation. 2000;101:790-6.

22. Cain BS, Meldrum DR, Joo KS, Wang JF, Meng X, Cleveland JC Jr, et al. Human SERCA2a levels correlate inversely with age in senescent human myocardium. J Am Coll Cardiol. 1998;32:458-67.

23. Rakusan K, Nagai J. Morphometry of arterioles and capillaries in hearts of senescent mice. Cardiovasc Res. 1994;28:969-72.

24. Rivard A, Fabre JE, Silver M, Chen D, Murohara T, Kearney M, Magner M, et al. Age-dependent impairment of angiogenesis. Circulation. 1999;99:111-20. 